\title{
Adaptaciones musculares relacionadas a áreas corporales que participan activamente en el vuelo de Anhima cornuta (Aves: Anseriformes, Anhimidae)
}

\section{Related muscular adaptations to corporal regions that actively participate in the flight of Anhima cornuta (Aves: Anseriformes, Anhimidae)}

\author{
Marcos Antonio Manzanares
}

Coordinador de Apoyo Técnico. Fundación Nacional de Parques Zoológicos y Acuarios de Venezuela. Sede Ministerio del Ambiente y de los Recursos Naturales. Centro Simón Bolívar, Torre Sur, Piso 6, Oficina FUNPZA, EI Silencio, Apartado Postal 40028 Caracas 1040, Venezuela. ++58-212-408-2164. Email Marcos Manzanares: Mamanzana@hotmail.com

Presentado: $\quad 12 / 06 / 2006$ Aceptado: $\quad 18 / 02 / 2007$

\section{Resumen}

La forma y correspondencia entre la anatomía muscular y ósea revela en gran medida el hábito locomotor de cualquier ave. Las especies pertenecientes a la Familia Anhimidae son relevantes dentro de las Aves, debido a las adaptaciones óseas (y por lo tanto, musculares) que presentan, como la pérdida de procesos uncinados, el desarrollo de espolones metarcapales, la extrema neumatización y el notable desarrollo de forámenes. Se analizaron anatómicamente y numéricamente (superficie de origen e inserción, longitud en reposo y masa) los componentes musculares relacionados a las zonas de la columna vertebral, tronco, miembros y cinturas de tres ejemplares de Anhima cornuta de distintos sexos y se compararon con lo estudiado en 4 ejemplares de especies relacionadas (Dendrocygna bicolor y Dendrocygna viduata, Anatidae). El estudio reveló que en Anhima existe un gran desarrollo de los músculos intercostales e intervertebrales (torácicos y lumbares), evidenciando una adaptación a la ausencia de procesos uncinados. En Anhima existen fascículos proporcionalmente menos pesados y de menor tamaño que en Dendrocygna, indicando morfológicamente la ocurrencia de un vuelo menos desarrollado en el primero. Algunos tendones y fibras musculares metacarpales cuya inserción ocurre en el primer dígito en Dendrocygna, en Anhima se insertan en la base de los espolones proximal y distal, demostrando el poco control del vuelo en Anhima. La musculatura de los miembros posteriores en Anhima es mucho más desarrollada, tanto en tamaño, como en masa proporcional, evidenciando una gran actividad en esta zona en la propulsión del vuelo y en hábitos terrestres.

Palabras clave: Anhimidae, Anhima, Dendrocygna, Musculatura, Anseriformes.

\section{Abstract}

The form and correspondence between the muscular and bony anatomy shows, principally, the locomotive habit of the birds. The screamers are a peculiar group in the Aves Class, because their bony adaptations (and therefore, muscular adaptations), the absence of uncinate processes, two long and sharp metacarpal spurs, their bones are far more pneumatic than in others birds and the bony foramens development. Four specimens of Anhima cornuta and four specimens of linked species (two of Dendrocygna bicolor and two of Dendrocygna viduata) were studied and compared, and their muscular components of the spine, trunk, forelimbs, hind limbs, tail and waists were analyzed, anatomically and quantitatively (origin and insertion surface, long, mass). The study revealed that Anhima has a great development of the intercostals and intervertebrals muscles (thoracic and lumbar regions), evidencing an adaptation to the absence of uncinate processes. Anhima has muscular fascicles, proportionally less heavy and smaller that Dendrocygna, indicating a less capacity of the flight. Some metacarpal tendons and muscular fibers whose inserts in the first digit in Dendrocygna, in Anhima are inserted in the spurs base, showing the few flight control. The hind limbs musculature in Anhima is much more developed, in size and proportional mass, evidencing a great activity in this area, specifically in the flight propulsion and in terrestrial habits.

Keywords: Anhimidae, Anhima, Dendrocygna, Musculature, Anseriformes.

\section{Introducción}

Todos los animales, incluyendo a las aves, presentan una correspondencia entre la forma, la estructura de la anatomía y el tipo de locomoción que efectúan. Este principio de forma vinculada con la función tiene un fundamento evolutivo, pues son las presiones adaptativas las que influyen en el cambio de una estructura; más específicamente, son las presiones en la musculatura de un ave (por ejemplo, la locomoción) las que inducen a la determinación de la conformación ósea (Witmer, 1995). Un perfecto ejemplo de este postulado se puede encontrar en los Anhimidos.

La Familia Anhimidae reúne a tres especies de aves distribuidas en zonas húmedas de Sudamérica tropical y subtropical. Los Anhimidos se destacan por su alimentación herbívora, la presencia de lamelas vestigiales, la existencia de membranas interdigitales no completas, la ausencia de procesos uncinados, el desarrollo de forámenes óseos, la extrema neumatización de los huesos, la presencia de dos espolones metacarpales, entre otras características (del Hoyo et al, 1992; Gill, 1999; Gómez Dallmeier, 1989; Hilty, 2003; Phelps \& Meyer de Schauensee, 1979). Aunque varias de estas características corresponden a aves antiguas o no voladoras, los Anhimidos constituyen un taxón cuyos miembros realizan vuelos sostenidos, pero sin ser tan gráciles y diestros como los observados en aves filogenéticamente relacionadas, como los Anátidos.

Las diferencias morfológicas existentes en el sistema óseo entre los Anhimidos y los Anátidos indican una conformación 


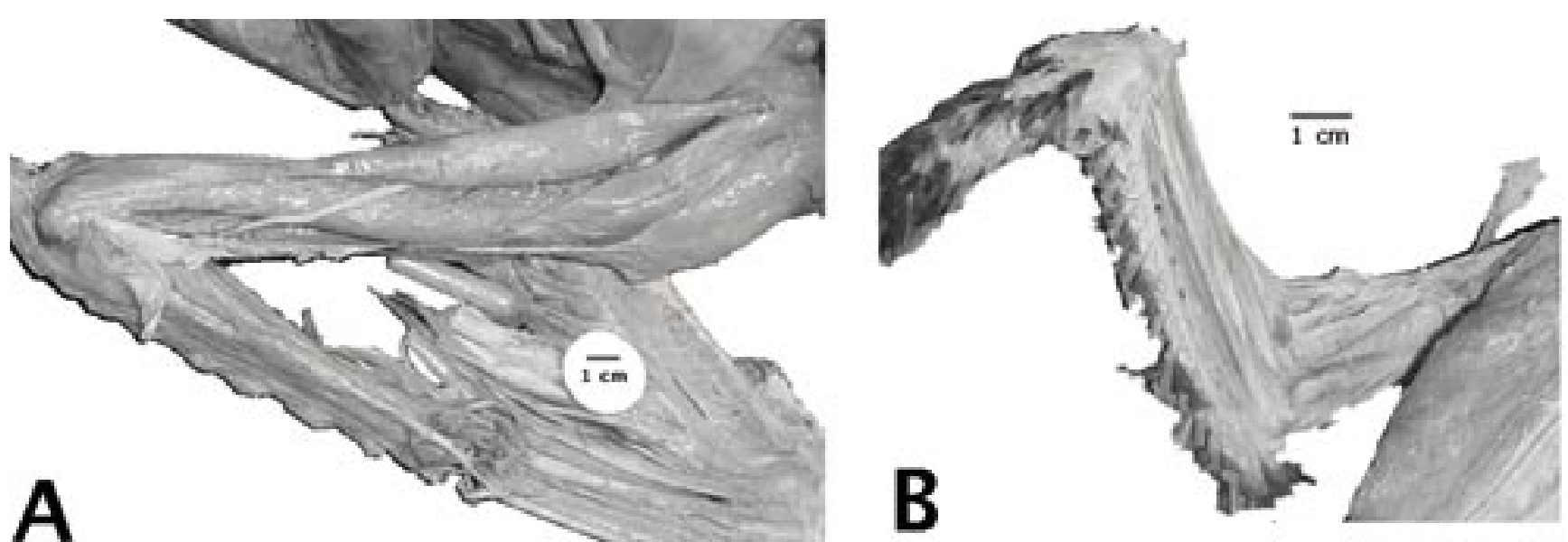

Figura 1. Detalle de la musculatura del antebrazo y del brazo derecho de Anhima cornuta (A) y Dendrocygna viduata (B). Observe la separación en diversos paquetes musculares de fascículos por fascias en A, y la importante agrupación muscular por una gruesa capa de tejido conjuntivo en $\mathrm{B}$.

muscular distinta, y por consiguiente, una variación sustancial en la realización del movimiento. Por lo tanto, la ejecución de un estudio sobre las adaptaciones musculares en Anhimidos permite efectuar un análisis de la conformación muscular y su efecto en el desarrollo del vuelo, además de contribuir en la investigación acerca de la evolución de los Anseriformes.

El siguiente trabajo representa un aporte a los escasos estudios de los Anhimidos, al combinar análisis de morfometría y morfología descriptiva con deducciones funcionales en locomoción, específicamente en la propulsión y desarrollo del vuelo en Anhima cornuta Linnaeus, 1766, comparada con otros Anseriformes, Dendrocygna bicolor Vieillot, 1816 y Dendrocygna viduata Linnaeus, 1766.

\section{Materiales y métodos}

Se preservaron en una solución 9:1:1 de alcohol etílico al $75 \%$, glicerina y formol al 5\%, tres especímenes de $A$. cornuta (dos hembras y un macho) y tres del género Dendrocygna (dos de $D$. bicolor y uno de $D$. viduata, un macho y una hembra para la primera especie y una hembra para la segunda), los cuales fueron capturados por inmovilización mecánica (mediante el uso de una escopeta semiautomática, calibre 16, perdigón \#8) en su hábitat: ejemplares de Dendrocygna en cultivos de arroz ubicados a $8 \mathrm{Km}$ al sur de Corozopando, Municipio Camaguán, Estado Guárico, Venezuela; un ejemplar de Anhima en un estero entre las localidades de El loco y Vuelta Redonda, Municipio José Antonio Páez, Estado Apure, Venezuela. Los otros dos ejemplares de Anhima -un macho y una hembra- fueron donados por el Parque Zoológico de Caricuao, en Caracas, Venezuela, y pertenecían a su colección de ejemplares preservados.

Se tomaron las medidas de longitud total del cuerpo, desde la cabeza hasta la base de la cola. De acuerdo con esta tarea se realizan las comparaciones de mediciones de extensión muscular. Posteriormente se desarrolló el análisis de descripción de fascículos en los sistemas eje - centro de gravedad - propulsión/ control; a saber: columna - región torácica - miembros/cinturas, empleando la terminología anatómica desarrollada por Pettingill (1970) y por Baumel et al. (1993). Se analizaron un total de 93 músculos, distribuidos en las siguientes zonas de acción común: 30 músculos en el antebrazo, 24 en el hombro y el brazo, 20 en el tronco y cola y 19 en la región vertebral. Las descripciones se realizaron basándose en dichos grupos, destacándose la disposición, origen, inserción, dirección de las fibras, diferencia de coloración, forma, relación con otros componentes (huesos, plumas, piel y otros músculos), y agrupación de fascículos por tejidos conjuntivos (fascias).

El estudio cuantitativo de la musculatura comprendió el análisis de la longitud total en reposo y masa muscular en seco, siguiendo la metodología empleada por Bodini (1977) en mamíferos (primates). Trece músculos fueron analizados cuantitativamente; su selección fue por su importancia en el movimiento de los miembros anteriores (Manzanares, 2003) y su conspicuidad. Los músculos evaluados son: Pectoralis major, Supracoracoideus, Coracobrachialis cranialis, Coracobrachialis caudalis, Scapulohumeralis, Subcoracoscapularis (pars subscapularis y pars subcoracoideus), Rhomboideus superficialis, Rhomboideus profundus, Extensor digitorum comunis, Deltoideus major, Deltoideus minor, Biceps brachii y Triceps brachii (pars scapulotriceps, pars humerotriceps y pars coracotriceps).

Por la imposibilidad de colección de un mayor número de ejemplares, los datos cuantitativos no fueron analizados a través de pruebas estadísticas paramétricas, sino por medias, desviaciones estándar, relaciones y porcentajes, comparando resultados entre géneros.

\section{Resultados}

La medición de longitud del cuerpo de los ejemplares de $A n$ hima dio como resultado 85,892 $\pm 4,343 \mathrm{~cm}$. En Dendrocygna, el resultado fue de $46,968 \pm 2,888 \mathrm{~cm}$.

Al evaluar la musculatura de Anhima y Dendrocygna, se observaron diferencias en la disposición, agrupación, forma y coloración. En la región del antebrazo, en Anhima existe una fuerte separación de músculos por una gruesa capa de tejido conjuntivo y a su vez, estos fascículos son agrupados por otra fascia. En Dendrocygna, este tejido resulta ser más delgado y menos resistentes al corte del bisturí (ver figura 1). Los músculos que son agrupados por este tejido realizan funciones similares (conjunto de fascículos encargados de la flexión, de la extensión, de la pronación, de la supinación, de la abducción y de la aducción de la zona del antebrazo y de la tensión del patangio y propatangio). La musculatura asociada a la región de la mano en Anhima (carpometacarpo y falanges) se encuentra fuertemente cubierta por este tejido, y se observó la inserción de fascículos en 

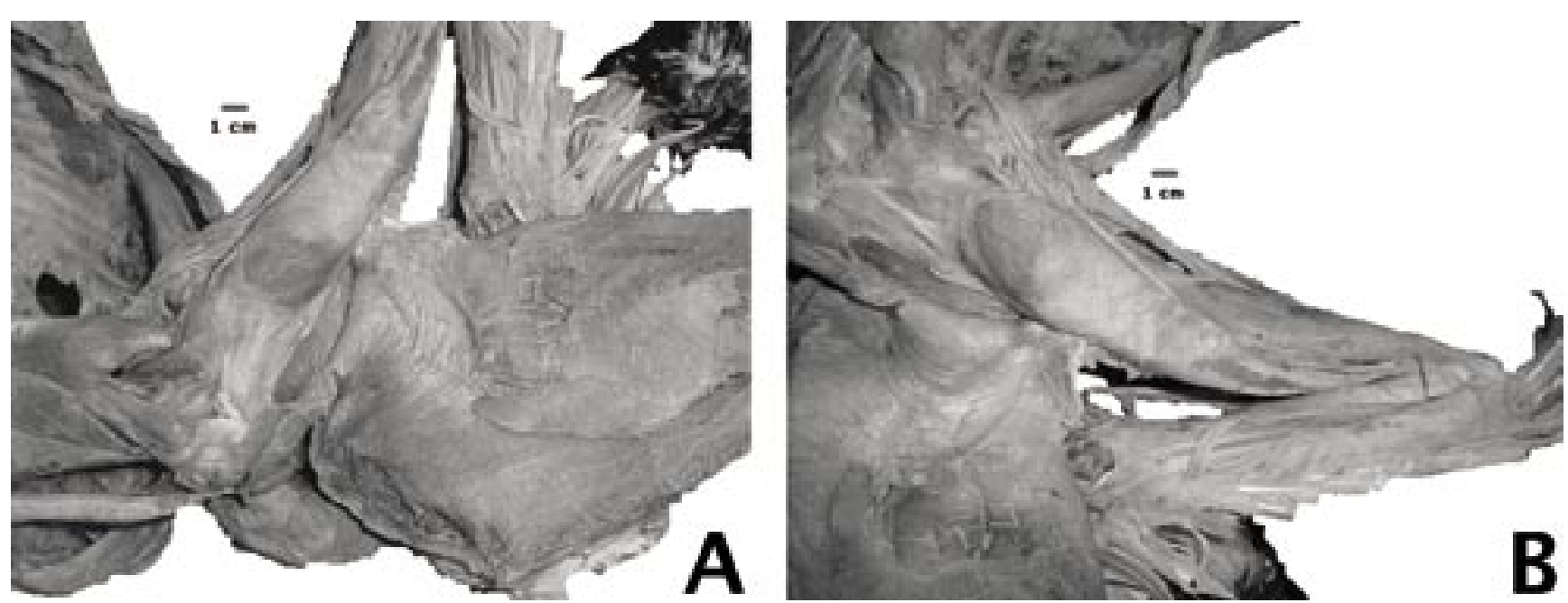

Figura 2. Musculatura de la región del hombro y del brazo izquierdo de Anhima cornuta (A) y Dendrocygna bicolor (B). Detalle la similitud en la agrupación muscular y el desarrollo de los fascículos en el hombro de $\mathrm{D}$. bicolor.

las bases de los espolones metacarpales. En el espolón metacarpal proximal (el de mayor tamaño) se insertan: Extensor digitorum comunis (pars alulae), Extensor metacarpi ulnaris, Abductor alulae, Adductor alulae, Flexor alulae y Extensor brevis alulae. La región de inserción muscular en la zona del álula y de este espolón es común. En la base del espolón metacarpal distal (el más pequeño) se insertan: Extensor digitorum comunis y Extensor metacarpi ulnaris, según la preparación.

En la región del hombro y brazo, en Anhima se observa que los músculos pectorales (Pectoralis major y Supracoracoideus) son anchos y planos (ver figuras 1A, 2A y 3B). En Dendrocygna, los músculos pectorales son alargados, engrosados cranealmente y de color similar al resto de la musculatura de la región (figura 2B). Los fascículos asociados a la región del brazo en Anhima son gruesos, compactos y sus fibras son resistentes al corte del bisturí. En cambio, en Dendrocygna, estos fascículos son alargados y en general, anchos en su origen (ocurre en los músculos Biceps brachii y Triceps brachii, ver figura 1B y 2B). Los fascículos situados en el hombro de Anhima son planos, fibrosos, anchos y con una coloración oscura, si se le compara con los presentados en Dendrocygna, donde estos se encuentran engrosados en su origen y son más alargados. Es de destacar la forma del músculo Deltoideus major, el cual en Anhima se presenta ancho y con dos proyecciones en su origen. En Dendrocygna, este músculo no presenta las proyecciones dichas y es plano en su origen. De igual forma al conjunto de músculos explicados anteriormente, se presenta la agrupación de fascículos que realizan funciones similares durante el desarrollo de la locomoción a través de tejido conjuntivo (músculos que participan en la elevación y descenso del húmero, en la abducción y aducción del brazo, en la rotación humeral, en la estabilización de la cintura escapulopectoral y en la tensión del patangio).

La musculatura de la región del tronco y de la cola de Anhima se caracteriza por ser plana, con grosor similar desde su origen a su inserción, de fibras resistentes y fuertes y de color oscuro en la zona más cercana a la caja torácica y esternón (figuras 2 a y
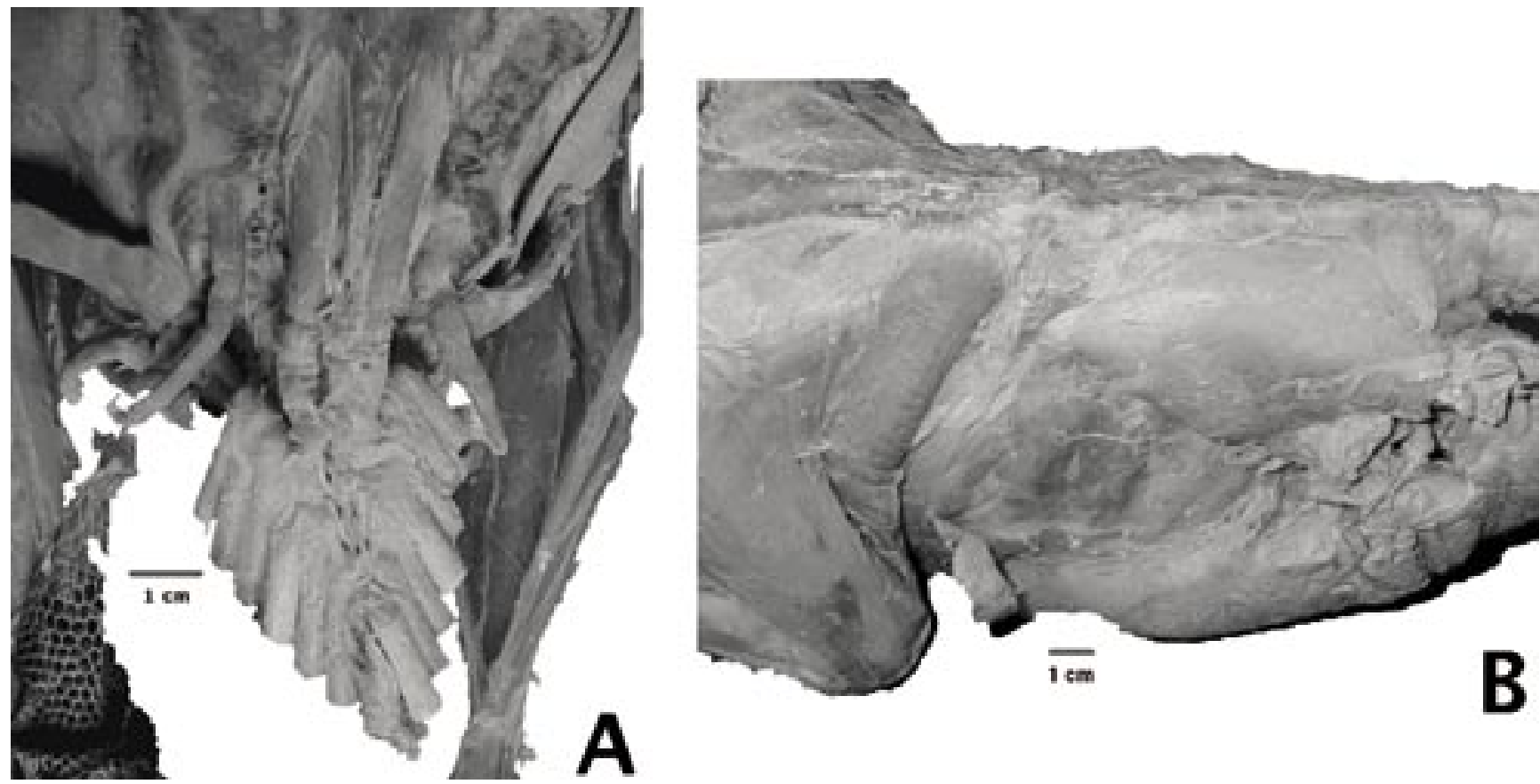

Figura 3. Detalle de la musculatura de la cola $(A)$ y el tronco $(B)$ de Anhima cornuta. Observe el desarrollo lateral de los músculos escapulares y separación de fascículos por fuertes fascias. 
Tabla 1. Longitud, peso y su respectiva relación, de los músculos que participan en la articulación humeral con la fosa glenoidea, tomados de tres ejemplares de Anhima cornuta. Se muestran valores promedio de longitud y peso, junto con la desviación estándar de cada valor. La relación de longitudes musculares corresponde al cociente entre la longitud muscular y longitud total corporal. La relación de pesos musculares corresponde al porcentaje que cada fascículo tiene con respecto al peso total de la musculatura de la región.

\begin{tabular}{|c|c|c|c|c|c|}
\hline & & \multicolumn{2}{|c|}{ Longitud Muscular } & \multicolumn{2}{|c|}{ Peso Muscular } \\
\hline & & $\begin{array}{l}\text { Longitud } \\
\text { Total }(\mathrm{cm})\end{array}$ & $\begin{array}{c}\text { Relación } \\
\text { Long Muscular } \\
\text { - Long Cuerpo }\end{array}$ & $\begin{array}{c}\text { Peso } \\
\text { Seco }(g)\end{array}$ & $\begin{array}{c}\text { Relación } \\
\text { Peso Muscular } \\
\text { - Peso Total (\%) }\end{array}$ \\
\hline \multicolumn{2}{|c|}{ Pectoralis major } & $12,88 \pm 1,23$ & 0,15 & $108,9 \pm 4,97$ & 71,57 \\
\hline \multicolumn{2}{|c|}{ Supracoracoideus } & $11,93 \pm 0,11$ & 0,14 & $8,2 \pm 3,08$ & 5,39 \\
\hline \multicolumn{2}{|c|}{ Coracobrachialis cranialis } & $3,77 \pm 0,06$ & 0,04 & $1,21 \pm 0,55$ & 0,80 \\
\hline \multicolumn{2}{|c|}{ Coracobrachialis caudalis } & $3,03 \pm 0,06$ & 0,04 & $0,99 \pm 0,03$ & 0,65 \\
\hline \multicolumn{2}{|c|}{ Scapulohumeralis } & $10,79 \pm 0,23$ & 0,13 & $4,63 \pm 0,05$ & 3,04 \\
\hline \multirow{2}{*}{$\begin{array}{c}\text { Subcoracoscapu- } \\
\text { lares }\end{array}$} & Subscapularis & $9,06 \pm 0,1$ & 0,11 & $4,51 \pm 1,16$ & 2,96 \\
\hline & Subcoracoideus & $7,26 \pm 0,35$ & 0,08 & $1,63 \pm 0,27$ & 1,07 \\
\hline \multicolumn{2}{|c|}{ Rhomboideus superficialis } & $9,11 \pm 0,15$ & 0,11 & $0,72 \pm 0,19$ & 0,47 \\
\hline \multicolumn{2}{|c|}{ Rhomboideus profundus } & $8,81 \pm 0,12$ & 0,10 & $0,9 \pm 0,21$ & 0,59 \\
\hline \multicolumn{2}{|c|}{ Extensor digitorum comunis } & $26,93 \pm 1,58$ & 0,31 & $2,46 \pm 0,03$ & 1,62 \\
\hline \multicolumn{2}{|c|}{ Deltoideus major } & $11,75 \pm 0,63$ & 0,14 & $3,32 \pm 0,15$ & 2,18 \\
\hline \multicolumn{2}{|c|}{ Deltoideus minor } & $4,59 \pm 0,62$ & 0,05 & $2,12 \pm 0,05$ & 1,39 \\
\hline \multicolumn{2}{|c|}{ Biceps brachii } & $18,86 \pm 4,48$ & 0,22 & $3,21 \pm 0,52$ & 2,11 \\
\hline \multirow{3}{*}{ Triceps brachii } & Scapulotriceps & $22,07 \pm 0,45$ & 0,26 & $4,09 \pm 0,01$ & 2,69 \\
\hline & Humerotriceps & $20,94 \pm 1,26$ & 0,24 & $3,96 \pm 0,04$ & 2,60 \\
\hline & Coracotriceps & $20,77 \pm 0,31$ & 0,24 & $1,31 \pm 0,15$ & 0,86 \\
\hline
\end{tabular}

3b). Se encuentra fuertemente agrupada por fascia los fascículos musculares intercostales y los dorsales (asociados a la escápula). Los músculos intercostales (Intercostales externus, Intercostales internus, Costosternalis, y Sternocoracoideus) son evidentes por su color rojo oscuro, su ancho y su gran resistencia al corte. Los músculos abdominales son gruesos y también son de color oscuro. En Dendrocygna, los músculos de esta región son anchos en su origen y se afinan en su inserción. Son de color claro, y su resistencia al corte es importante, aunque menor que en Anhima. La coloración es similar al resto de la musculatura de la región. La cola de Anhima presenta músculos alargados y delgados, de color claro en la superficie dorsal-medial y oscuros y fuertes en los laterales y en la región dorso-lateral de la misma. En Dendrocygna, estos músculos son más pequeños y muy claros. Esto se evidencia en el desarrollo de la cola de los ejemplares de ambos géneros, siendo más larga y ancha en Anhima, y más corta y en forma de triángulo en Dendrocygna.

Los músculos de la región vertebral de Anhima son similares en coloración y desarrollo que los presentados en Dendrocygna, a diferencia del notable grosor de los músculos de las vértebras torácicas, lumbares y sacras en el primero, y el desarrollo de los músculos cervicales en el segundo.

Los resultados del análisis cuantitativo de los músculos que participan en la articulación del húmero con la fosa glenoidea, tanto en los ejemplares de Anhima como en los ejemplares de Dendrocygna, se muestran en las tablas 1 y 2 , respectivamente.

Respecto a la longitud, los músculos más notables en Dendrocygna corresponden a los que participan en la propulsión del vuelo (Pectoralis major y Supracoracoideus). En cambio, en Anhima se observaron que los músculos que participan en la elevación, descenso y flexión del húmero, así como en la estabilización de la cintura escapulopectoral son los más largos (Coracobrachialis cranialis, Coracobrachialis caudalis, Rhomboideus superficialis, Rhomboideus profundus y Triceps brachii).

Con respecto a las mediciones de peso seco, los músculos más evidentes en Dendrocygna son los que participan en la elevación y descenso del húmero (Coracobrachialis cranialis, Coracobrachialis caudalis, Pectoralis major, Supracoracoideus y Triceps brachii pars scapulotriceps). En Anhima, los que presentan mayor peso son los encargados de estabilizar la cintura escapulopectoral, mantener la rigidez de esta zona y desarrollar los movimientos de húmero en la fosa glenoidea (Scapulohumeralis, Subcoracoscapularis, Rhomboideus superficialis, Rhomboideus profundus, Extensor digitorum comunis, Deltoideus major, Deltoideus minor y Triceps brachii -pars humerotriceps y pars coracotriceps-).

\section{Discusión}

Las especies estudiadas, a pesar de su vinculación sistemática, presentan modelos morfológicos distintos, y por ende, desarrollan locomociones diferentes. Comparando con el tipo anatómico presentado en Dendrocygna, Anhima presenta reducidos fascículos musculares en la región pectoral y en la zona proximal del húmero, indicando un menor desarrollo del vuelo activo, producido por el batido constante de los miembros anteriores (elevación y descenso del brazo). Aunado a los datos cuantitativos, las observaciones de morfología comparada indican que, por la coloración, no se presenta alta actividad mecánica en los músculos pectorales. La forma que tienen estos músculos en Anhima, los cuales se presentan como fascículos aplanados y extendidos lateralmente, puede indicar cierta participación en la rigidez de la caja torácica durante el vuelo.

El desarrollo (en peso, tamaño y disposición) de los fascículos que participan en los movimientos de abducción, aducción y 
rotación del húmero en Anhima, así como los que actúan en la estabilización y rigidez de su cintura escapulopectoral, indican una compensación con respecto a los músculos de la región pectoral. Por otra parte, la presencia de gruesas y fuertes fascias rodeando los músculos de esta región señala la ocurrencia de fuerzas de gran magnitud en los respectivos ejes de actividad, permitiendo así efectuar de mejor manera los movimientos de los miembros anteriores durante la locomoción y conferir rigidez a la cintura escapulopectoral durante el vuelo.

Es importante destacar los músculos Extensor digitorum comunis y Triceps brachii (pars Humerotriceps y pars Coracotriceps), los cuales actúan en la flexión y extensión del codo y en el movimiento conjunto del brazo y del antebrazo. Su desarrollo (en peso) en Anhima permite deducir una alta actividad, que puede no estar relacionada con su hábito locomotor, sino más bien por una característica morfológica presente en los Anhimidos, y que son los espolones metacarpales. Los Anhimidos suelen emplear sus espolones como defensa ante posibles depredadores y ante sus congéneres. Para ello, flexionan el codo, levantando el antebrazo y exponiendo el borde de los espolones (Rumboll, 1975). Este movimiento, que resulta ser constante en grupos libres de Anhimidos (Johnsgard, 1976; Manzanares, 2003), así como en otros grupos de aves acuáticas con espolones (Delacour, 1954, 1964; Johnsgard, y Carbonell, 1996), exige una gran actividad de los músculos que participan en la flexión y movimiento del antebrazo, permitiendo su desarrollo en peso y extensión.

Si bien los espolones metacarpales son empleados para la defensa de los Anhimidos, su presencia afecta el desarrollo del vuelo, en especial, su maniobrabilidad. Los músculos que participan en la locomoción del antebrazo, en especial, los que efectúan los movimientos intrínsecos (supinación y pronación), son indispensables para ejecutar el control durante el vuelo (Dial et al. 1997; Gatesy y Dial, 1996; Pennycuik, 1972). La presencia de los espolones metacarpales en Anhima afecta la disposición de los fascículos musculares de esta zona, al variar la ubicación de la superficie de inserción, modificando su actividad. Como apoyo a esta hipótesis, observaciones del movimiento del miembro anterior de Anhima cornuta realizadas durante el vuelo demuestran su poca maniobrabilidad ante obstáculos y al momento del aterrizaje (Manzanares, 2003).

En el eje vertebral, el desarrollo muscular en el tronco de Anhima (músculos vertebrales torácicos, lumbares y sacrales) revela la importante acción de sostén en la zona que efectúan durante la locomoción. Esta se ve complementada con la participación de los músculos torácicos y abdominales (Intercostales externus, Intercostales internus, Obliquus abdominis externus, Obliquus abdominis internus, Transversus abdominis, Costosternalis, Scalenus y Levatores costarum), los cuales se encuentran notablemente desarrollados y su coloración oscura indica alta actividad. Este desarrollo ocurre como respuesta evolutiva a la pérdida de rigidez de la caja torácica (como consecuencia de la pérdida de los procesos uncinados) y al elevado peso con relación al tamaño de los Anhimidos. Por ser aves herbívoras, los Anhimidos generalmente emprenden vuelo con gran cantidad de alimento en el tracto digestivo, por lo que la propulsión ocurre con un fuerte batido de alas y movimientos de los miembros posteriores (Manzanares, 2003), evidenciado en el desarrollo de los músculos de esta zona y de la cintura pélvica. Por lo tanto, la conformación muscular presentada en Anhima cornuta revela sus adaptaciones ante la pérdida de proyecciones osificadas en las costillas (procesos uncinados), la presencia de espolones metacarpales y su dieta estrictamente herbívora, exponiendo a su vez los efectos que tienen estas características morfológicas en el desarrollo de la locomoción.

Tabla 2. Longitud, peso y su respectiva relación, de los músculos que participan en la articulación humeral con la fosa glenoidea, tomados de tres ejemplares de Dendrocygna. Se muestran valores promedio de longitud y peso, junto con la desviación estándar de cada valor. La relación de longitudes musculares corresponde al cociente entre la longitud muscular y longitud total corporal. La relación de pesos musculares corresponde al porcentaje que cada fascículo tiene con respecto al peso total de la musculatura de la región.

\begin{tabular}{|c|c|c|c|c|c|}
\hline & & \multicolumn{2}{|c|}{ Longitud Muscular } & \multicolumn{2}{|c|}{ Peso Muscular } \\
\hline & & $\begin{array}{l}\text { Longitud } \\
\text { Total (cm) }\end{array}$ & $\begin{array}{c}\text { Relación } \\
\text { Long Muscular } \\
\text { - Long Cuerpo }\end{array}$ & $\begin{array}{c}\text { Peso } \\
\operatorname{Seco}(\mathrm{g})\end{array}$ & $\begin{array}{c}\text { Relación } \\
\text { Peso Muscular } \\
\text { - Peso Total (\%) }\end{array}$ \\
\hline \multicolumn{2}{|c|}{ Pectoralis major } & $10,09 \pm 1$ & 0,21 & $29,95 \pm 7,45$ & 78,40 \\
\hline \multicolumn{2}{|c|}{ Supracoracoideus } & $9,02 \pm 0,83$ & 0,19 & $2,26 \pm 0,65$ & 5,92 \\
\hline \multicolumn{2}{|c|}{ Coracobrachialis cranialis } & $1,49 \pm 0,18$ & 0,03 & $0,37 \pm 0,17$ & 0,97 \\
\hline \multicolumn{2}{|c|}{ Coracobrachialis caudalis } & $1,27 \pm 0,1$ & 0,03 & $0,41 \pm 0,04$ & 1,07 \\
\hline \multicolumn{2}{|c|}{ Scapulohumeralis } & $5,95 \pm 0,42$ & 0,13 & $0,72 \pm 0,21$ & 1,88 \\
\hline \multirow{2}{*}{ Subcoracoscapulares } & Subscapularis & $4,98 \pm 0,13$ & 0,11 & $0,08 \pm 0,03$ & 0,21 \\
\hline & Subcoracoideus & $3,88 \pm 0,11$ & 0,08 & $0,09 \pm 0,02$ & 0,24 \\
\hline \multicolumn{2}{|c|}{ Rhomboideus superficialis } & $3,76 \pm 0,12$ & 0,08 & $0,1 \pm 0,03$ & 0,26 \\
\hline \multicolumn{2}{|c|}{ Rhomboideus profundus } & $2,45 \pm 0,11$ & 0,05 & $0,19 \pm 0,04$ & 0,50 \\
\hline \multicolumn{2}{|c|}{ Extensor digitorum comunis } & $13,98 \pm 0,66$ & 0,30 & $0,28 \pm 0,08$ & 0,73 \\
\hline \multicolumn{2}{|c|}{ Deltoideus major } & $5,97 \pm 0,69$ & 0,13 & $0,63 \pm 0,18$ & 1,65 \\
\hline \multicolumn{2}{|c|}{ Deltoideus minor } & $3,09 \pm 0,19$ & 0,07 & $0,17 \pm 0,09$ & 0,45 \\
\hline \multicolumn{2}{|c|}{ Biceps brachii } & $9,96 \pm 0,03$ & 0,21 & $0,79 \pm 0,28$ & 2,07 \\
\hline \multirow{3}{*}{ Triceps brachii } & Scapulotriceps & $10,11 \pm 0,26$ & 0,22 & $1,32 \pm 0,56$ & 3,46 \\
\hline & Humerotriceps & $9,44 \pm 0,6$ & 0,20 & $0,67 \pm 0,12$ & 1,75 \\
\hline & Coracotriceps & $9,08 \pm 0,42$ & 0,19 & $0,17 \pm 0,04$ & 0,45 \\
\hline
\end{tabular}




\section{Agradecimientos}

Agradezco la disposición del Lic. William Bermúdez y Lic. Salvador Boher, por su colaboración en la recolección de datos y su amplia contribución para este trabajo. Asimismo, agradezco las facilidades brindadas por la Escuela de Biología de la Facultad de Ciencias - Universidad Central de Venezuela (UCV), para la elaboración de este trabajo. Agradezco también a la Fundación Nacional de Parques Zoológicos y Acuarios de Venezuela por permitirme emplear parte de sus instalaciones para la elaboración y análisis del manuscrito. Por último, agradezco muy especialmente al Comité Organizador del VI Congreso Nacional de Ornitología (VICNO) y a la Srta. Fabiola Riva, por su excelente organización y trato, su constante apoyo y sus palabras de aliento para la publicación de este trabajo científico y del resto de los trabajos de mis colegas.

\section{Literatura citada}

Baumel, J. J., A. S. King, J. E. Breazile, H. E. Evans \& J. C. Vanden Berge, eds. 1993. Handbook of Avian Anatomy. Nomina Anatomica Avium. Second Edition. Publication of the Nuttall Ornithological Club 23. Harvard University, Cambridge, MA. 779 pp.

Bodini, R. 1977. Análisis de las funciones de la musculatura del miembro de la cadera de los Cebidae. Aporte metódico y funcional a la biomecánica del miembro posterior de los primates. Trabajo de Ascenso, Facultad de Ciencias, Escuela de Biología, Departamento de Zoología, Universidad Central de Venezuela, Caracas, Venezuela.

Del Hoyo, J., A. Elliot \& J. Sargatal, eds. 1992. Handbook of the birds of the world. Vol. 1. Ostrich to Ducks. ICBP LYNX Edition, Barcelona, España. Pp. 528-627.

Delacour, J. 1954. The Waterfowls of the world. Vol. 1. Country Life Limited. London, England. Pp. 15-49.

Delacour, J. 1964. The Waterfowls of the world. Vol. 4. Country Life Limited. London, England. Pp. 108-326.
Dial, K. P.; A. A. Biewener; B. W. Tobalske \& D. R. Warrick. 1997. Mechanical power output of bird flight. Nature. Vol. 390. Pp. $67-70$.

Gatesy, S. M. \& K. P. Dial. 1996. Locomotor modules and the evolution of avian flight. Evolution, Vol. 50 (1). Pp. 331-340.

Gill, F. 1994. Ornithology. Second Edition. W. H. Freeman and Co. New York. 758 pp.

Gómez-Dallmeier, F. \& A. T. Cringan. 1989. Biology conservation and management of waterfowl in Venezuela. Editorial Ex Libris, Caracas, Venezuela.

Hilty, S. L. 2003. Birds of Venezuela. Second Edition. Princeton University Press, 41 William Street, Princeton, New Jersey 08540. 878 Pp.

Johnsgard, P. A. 1976. Handbook of waterfowl behavior. Cornell University Press. Ithaca, New York. 378pp.

Johnsgard, P. A. \& M. Carbonell. 1996. Ruddy ducks and other stifftails. Their behavior and biology. University of Oklahoma Press, Norman, USA

Manzanares, M. 2003. Estudio comparativo de los componentes musculares, esqueléticos y tegumentarios asociados al vuelo en el Aruco, Anhima cornuta, y en el Yaguazo Colorado, Dendrocygna bicolor (Aves: Anseriformes). 146 páginas. Trabajo especial de grado. Licenciatura de Biología. Universidad Central de Venezuela, Caracas, Venezuela.

Pennycuik, C. J. 1972. Animal flight. Studies in Biology. No. 33. Arnold, London.

Pettingill, O. S. Jr. 1970. Ornithology in Laboratory and Field. Fourth Edition. Burgess Publishing Company. Minneapolis, Minnesota. 524pp.

Phelps, W. H. \& Meyer de Schauensee. 1979. Una guía de Aves de Venezuela. Gráficas Armitano.

Rumboll, M. A. E. 1975. Espolones metacarpales del Chajá (Chauna torquata). Hornero 11: 316-317.

Witmer, L. M. 1995. The extant phylogenetic bracket and the importance of reconstructing soft tissues in fossilis. In: J. Thomason, ed. Functional Morphology in Vertebrate Paleonthology. Pp. 19-33. Cambridge University Press. 